2013-6

\title{
Novel Dielectric Loaded Plasmonic Waveguide for Tight-Confined Hybrid Plasmon Mode
}

\author{
Youqiao Ma \\ Technological University Dublin \\ Gerald Farrell \\ Technological University Dublin, gerald.farrell@tudublin.ie \\ Yuliya Semenova \\ Technological University Dublin, yuliya.semenova@tudublin.ie
}

See next page for additional authors

Follow this and additional works at: https://arrow.tudublin.ie/engscheceart

\section{Recommended Citation}

Ma, Y., Farrell, G., Semenova, Y., Chan, H., Zhang, H. and Wu, Q. Novel dielectric loaded plasmonic waveguide for tight-confined hybrid plasmon mode. Plasmonics, vol. 8, pp. 1259-1263, 2013. DOI: 10.1007/s11468-013-9543-9.

This Article is brought to you for free and open access by the School of Electrical and Electronic Engineering at ARROW@TU Dublin. It has been accepted for inclusion in Articles by an authorized administrator of ARROW@TU Dublin. For more information, please contact arrow.admin@tudublin.ie, aisling.coyne@tudublin.ie, gerard.connolly@tudublin.ie.

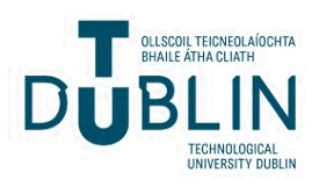




\section{Authors}

Youqiao Ma, Gerald Farrell, Yuliya Semenova, Hauping Chan, Hongzhou Zhang, and Qiang wu 


\section{Dear Author}

Here are the proofs of your article.

- You can submit your corrections online, via e-mail or by fax.

- For online submission please insert your corrections in the online correction form. Always indicate the line number to which the correction refers.

- You can also insert your corrections in the proof PDF and email the annotated PDF.

- For fax submission, please ensure that your corrections are clearly legible. Use a fine black pen and write the correction in the margin, not too close to the edge of the page.

- Remember to note the journal title, article number, and your name when sending your response via e-mail or fax.

- Check the metadata sheet to make sure that the header information, especially author names and the corresponding affiliations are correctly shown.

- Check the questions that may have arisen during copy editing and insert your answers/corrections.

- Check that the text is complete and that all figures, tables and their legends are included. Also check the accuracy of special characters, equations, and electronic supplementary material if applicable. If necessary refer to the Edited manuscript.

- The publication of inaccurate data such as dosages and units can have serious consequences. Please take particular care that all such details are correct.

- Please do not make changes that involve only matters of style. We have generally introduced forms that follow the journal's style.

- Substantial changes in content, e.g., new results, corrected values, title and authorship are not allowed without the approval of the responsible editor. In such a case, please contact the Editorial Office and return his/her consent together with the proof.

- If we do not receive your corrections within 48 hours, we will send you a reminder.

- Your article will be published Online First approximately one week after receipt of your corrected proofs. This is the official first publication citable with the DOI. Further changes are, therefore, not possible.

- The printed version will follow in a forthcoming issue.

\section{Please note}

After online publication, subscribers (personal/institutional) to this journal will have access to the complete article via the DOI using the URL:

http://dx.doi.org/10.1007/s11468-013-9543-9

If you would like to know when your article has been published online, take advantage of our free alert service. For registration and further information, go to:

http://www.springerlink.com.

Due to the electronic nature of the procedure, the manuscript and the original figures will only be returned to you on special request. When you return your corrections, please inform us, if you would like to have these documents returned. 


\section{Metadata of the article that will be visualized in OnlineFirst}

Please note: Images will appear in color online but will be printed in black and white.

\begin{tabular}{|c|c|c|c|}
\hline 1 & Article Title & \multicolumn{2}{|c|}{$\begin{array}{l}\text { Novel Dielectric-Loaded Plasmonic Waveguide for Tight- } \\
\text { Confined Hybrid Plasmon Mode }\end{array}$} \\
\hline 2 & Article Sub-Title & & \\
\hline 3 & $\begin{array}{l}\text { Article Copyright - } \\
\text { Year }\end{array}$ & \multicolumn{2}{|c|}{$\begin{array}{l}\text { Springer Science+Business Media New York } 2013 \\
\text { (This will be the copyright line in the final PDF) }\end{array}$} \\
\hline 4 & Journal Name & Plasmonics & \\
\hline 5 & \multirow{8}{*}{$\begin{array}{l}\text { Corresponding } \\
\text { Author }\end{array}$} & Family Name & $\mathbf{W u}$ \\
\hline 6 & & Particle & \\
\hline 7 & & Given Name & Qiang \\
\hline 8 & & Suffix & \\
\hline 9 & & Organization & Dublin Institute of Technology \\
\hline 10 & & Division & $\begin{array}{l}\text { Photonics Research Center, School of Electronic } \\
\text { and Communications Engineering }\end{array}$ \\
\hline 11 & & Address & Kevin Street, Dublin 8, Ireland \\
\hline 12 & & e-mail & Qiang.wu@dit.ie \\
\hline 13 & \multirow{8}{*}{ Author } & Family Name & Ma \\
\hline 14 & & Particle & \\
\hline 15 & & Given Name & Youqiao \\
\hline 16 & & Suffix & \\
\hline 17 & & Organization & Dublin Institute of Technology \\
\hline 18 & & Division & $\begin{array}{l}\text { Photonics Research Center, School of Electronic } \\
\text { and Communications Engineering }\end{array}$ \\
\hline 19 & & Address & Kevin Street, Dublin 8, Ireland \\
\hline 20 & & e-mail & \\
\hline 21 & \multirow{8}{*}{ Author } & Family Name & Farrell \\
\hline 22 & & Particle & \\
\hline 23 & & Given Name & Gerald \\
\hline 24 & & Suffix & \\
\hline 25 & & Organization & Dublin Institute of Technology \\
\hline 26 & & Division & $\begin{array}{l}\text { Photonics Research Center, School of Electronic } \\
\text { and Communications Engineering }\end{array}$ \\
\hline 27 & & Address & Kevin Street, Dublin 8, Ireland \\
\hline 28 & & e-mail & \\
\hline
\end{tabular}




\begin{tabular}{llll}
29 & Family Name & Semenova \\
30 & Particle & \\
31 & Given Name & Yuliya \\
32 & Suffix & \\
33 & Author & Organization & Dublin Institute of Technology \\
34 & Division & $\begin{array}{l}\text { Photonics Research Center, School of Electronic } \\
\text { and Communications Engineering }\end{array}$ \\
35 & & Address & Kevin Street, Dublin 8, Ireland \\
36 & e-mail & \\
37 & Family Name & Chan \\
38 & Particle & \\
39 & Given Name & Hau Ping \\
40 & Suffix & \\
41 & Author & Organization & City University of Hong Kong \\
42 & Division & Department of Electronic Engineering \\
43 & Address & Hong Kong, China \\
44 & e-mail & \\
\hline 45 & Family Name & Zhang \\
46 & Particle & \\
47 & Given Name & Hongzhou \\
48 & Suffix & \\
49 & Organization & Trinity College Dublin \\
50 & Author & Division & School of Physics \\
51 & Address & Dublin 2, Ireland \\
52 & Organization & Trinity College \\
53 & Division & CRANN \\
54 & Address & Dublin 2, Ireland \\
55 & e-mail & \\
\hline 56 & Received & 22 December 2012 \\
57 & Schedule & Revised & \\
58 & Accepted & 4 March 2013 \\
\hline & Abstract & In this letter, a novel metal-dielectric waveguide structure is \\
& proposed to support hybrid long range surface plasmon polaritons \\
& with a highly confined mode field. The simulation results showed \\
& that our proposed structure has better mode confinement and \\
& propagation length compared to that of conventional dielectric- \\
& loaded surface plasmon polaritons waveguides. This structure offers \\
& greater flexibility for the design of SPPs waveguides by altering the \\
& trade-off between mode confinement and propagation length. The \\
& proposed structure has significant potential for application in highly
\end{tabular}


integrated photonic circuits.

60 Keywords Surface plasmon resonant - Optical waveguide - Dielectric-loaded separated by ' - ' plasmonic waveguide

61 Foot note information 


\author{
Youqiao Ma • Gerald Farrell • Yuliya Semenova •

\begin{abstract}
In this letter, a novel metal-dielectric waveguide structure is proposed to support hybrid long range surface plasmon polaritons with a highly confined mode field. The simulation results showed that our proposed structure has better mode confinement and propagation length compared to that of conventional dielectric-loaded surface plasmon polaritons waveguides. This structure offers greater flexibility for the design of SPPs waveguides by altering the trade-off between mode confinement and propagation length. The proposed structure has significant potential for application in highly integrated photonic circuits.
\end{abstract}

Keywords Surface plasmon resonant - Optical waveguide . Dielectric-loaded plasmonic waveguide

In this paper, a novel metal-dielectric waveguide structure is proposed to support hybrid long range surface plasmon polaritons (LRSPPs) with a highly confined mode field.

Y. Ma $\cdot$ G. Farrell $\cdot$ Y. Semenova $\cdot$ Q. Wu $(\bowtie)$

Photonics Research Center, School of Electronic

and Communications Engineering, Dublin Institute of Technology,

Kevin Street, Dublin 8, Ireland

e-mail: Qiang.wu@dit.ie

H. P. Chan

Department of Electronic Engineering,

City University of Hong Kong, Hong Kong, China

H. Zhang

School of Physics, Trinity College Dublin, Dublin 2, Ireland

H. Zhang

CRANN, Trinity College, Dublin 2, Ireland
The simulation results showed that our proposed structure has better mode confinement and propagation length compared to that of conventional dielectric-loaded surface plasmon polaritons (DLSPPs) waveguides. This structure offers greater flexibility for the design of surface plasmon polaritons (SPPs) waveguides by altering the trade-off between mode confinement and propagation length. The proposed structure has significant potential for application in highly integrated photonic circuits.

The future development of optical communication systems will drive a requirement for high-density integration of photonics devices [1]. SPPs, which are electromagnetic waves (EMs) coherently coupled to electron oscillations and propagating at the interface between a dielectric and a metal conductor, can confine EMs at the subwavelength scale beyond the diffraction limit $[2,3]$ and therefore become ideal candidates for light guiding and confining in highly integrated optical circuits [4]. Recently, various plasmonic waveguides which provide nanoscale confinement have been proposed and investigated, such as a metal-insulator-metal waveguide [5], V-groove channel [6], and plasmonic wire waveguide [7]. These waveguides can significantly enhance the field confinement, but the associated decrease in the propagation length due increased absorption is often unacceptable. To achieve a long propagation length, the confinement will have to be relaxed but this will impact on the potential for high density integration. For example, in so-called long-range SPPs (LRSPPs), where guiding takes place by a thin metal film embedded in infinite homogeneous background dielectrics, it is possible to achieve a low propagation loss over a propagation length of a few millimeters [8]. However, as the LRSPPs mode size is in the order of a few micrometers, which causes significant bend loss in structures with tight 
bends or small radii with the optimal bend radius is as large as $10 \mathrm{~mm}$ [9], so that such devices are not suitable for high density photonic integration.

In order to optimize the tradeoff between the mode confinement and propagation length for SPPs [10], a DLSPPs waveguide with a reduced mode size (effective mode size of $1 \mu \mathrm{m})$ and a moderate propagation length at telecom wavelengths were recently proposed and shown to support a hybrid LRSPPs mode[11, 12]. The DLSPPs structure is very attractive as a plasmonic waveguide as it enables easy access to metal electrodes and thus offers the possibility of inducing electro- or thermo-optic effects in the waveguide components [12]. Recently, a hybrid LRSPPs waveguide consisting of a narrow metal film surrounded by low index dielectric regions has been theoretically analyzed, demonstrating a doubling of the propagation length $(\sim 756 \mu \mathrm{m})$ compared to that of a conventional structure $(\sim 356 \mu \mathrm{m})$ at expense of weaker mode confinement (i.e., the mode size increases $20 \%$ ) [13].

In this paper, a novel hybrid plasmonic waveguide offering lower loss but with a higher degree of mode confinement is proposed and investigated. The waveguide utilizes the dielectric configuration of DLSPPs but with an altered metallic structure to give a longer propagation length for the same mode confinement and a tighter mode confinement for the same propagation length, which offers greater flexibility in the design of SPPs waveguides by altering the trade-off between mode confinement and propagation length and hence has significant application potential in highly integrated photonic circuits.

Figure 1 shows a cross-sectional view of the proposed plasmonic waveguide, which consists of a thin metal film (with thickness $d$ and width $g$ in the central region, and thickness $h$ at both sides) symmetrically embedded inside a dielectric ridge with thickness $H$ and width $G$ surrounded by air. For such a symmetrical configuration, the index matching condition is satisfied so that a hybrid LRSPPs mode is supported. As shown in Fig. 1, the dielectric material is chosen to be $\mathrm{Si}_{3} \mathrm{~N}_{4}$, which has been commonly used by other authors. This dielectric material has a refractive index of $n_{\mathrm{r}}=2.0$, and gold is used as the metal with refractive index $n_{m}=0.55+11.5 i$ at the optical communication wavelength of $\lambda_{0}=1550 \mathrm{~nm}$. In the numerical simulations, the finite element method [14] is adopted to analyze the characteristics of the hybrid LRSPPs mode. The propagation length of the hybrid LRSPPs mode is calculated as $L_{p}$ $=\lambda_{o} /\left[4 \pi \operatorname{Im}\left(n_{\text {eff }}\right)\right]$, where $\operatorname{Im}\left(n_{\text {eff }}\right)$ is the imaginary part of the complex effective mode index $n_{\text {eff. }}$. The key parameter demonstrating the mode confinement capability is the normalized mode area $S$, which is defined by the formula $S=$ $S_{\text {eff }} / S_{0}$. Here, $S_{0}$ is the diffraction limited mode area $\left(S_{0}\right.$ $=\lambda_{0}{ }^{2} / 4$ ), and the effective mode area $S_{\text {eff }}$ is calculated using the following equations [15]:

$$
S_{\text {eff }}=\left(\iint W(\vec{r}) d S\right)^{2} /\left(\iint W(\vec{r})^{2} d S\right)
$$

$$
W(\vec{r})=\frac{1}{2} \operatorname{Re}\left\{\frac{d[w \varepsilon(\vec{r})]}{d w}\right\}|E(\vec{r})|^{2}+\frac{1}{2} u_{0}|H(\vec{r})|^{2}
$$

where $r$ is the coordinate, $\omega$ is angular frequency of the incident light, $|E(\vec{r})|^{2}$ and $|E(\vec{r})|^{2}$ are the intensities of electric and magnetic fields, respectively, $\varepsilon(\overrightarrow{2})$ is the dielectric permittivity and $\mu_{0}$ is the vacuum magnetic permeability.

Firstly, the relationship between the hybrid LRSPPs mode and the gold film thickness $h$ was investigated for a conventional DLSPP plasmonic waveguide (i.e., $d=h$ in Fig. 1). Figure 2 gives the calculated $L_{p}$ and $S$ versus gold thickness.

As shown in Fig. 2, both the propagation length $L_{p}$ and normalized mode area $S$ of the hybrid LRSPPs mode increases as the thickness of the metal film decreases. As expected, since a larger normalized mode area corresponds to a lower degree mode confinement [15], there is a trade-off between the mode confinement and propagation length. For example, increasing the metal film thickness can result in a better field confinement, but will introduce higher transmission loss. Conversely a decrease in the metal film thickness can enhance the propagation length, but will degrade the mode confinement. In order to improve the trade-off conditions, e.g., to achieve longer propagation length for the same

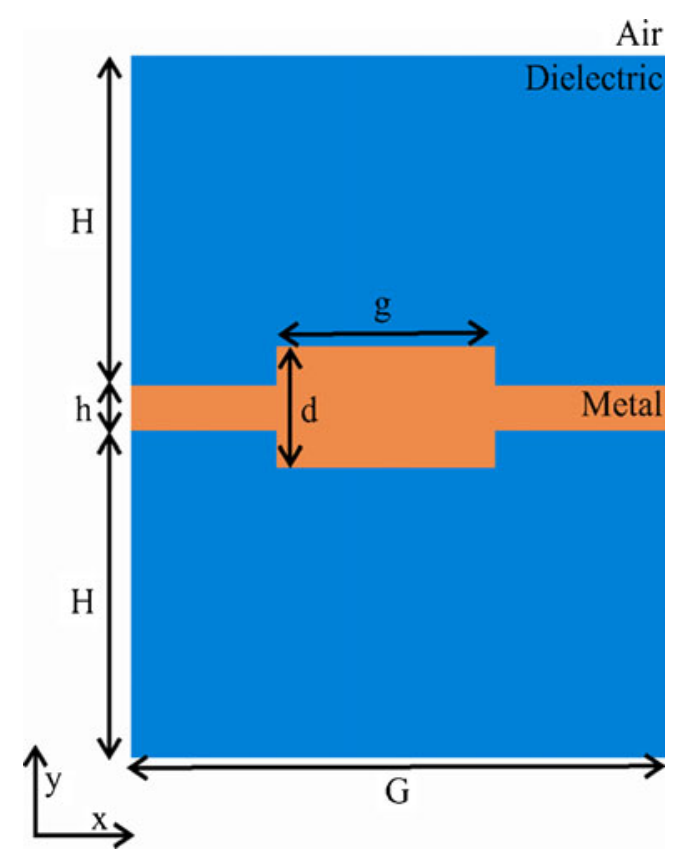

Fig. 1 (Color online) Schematic diagram of the cross-section of proposed DLSPPs waveguide. When $d$ equals to $h$, i.e., $d=h$, the structure becomes a conventional DLSPPs waveguide 
Plasmonics

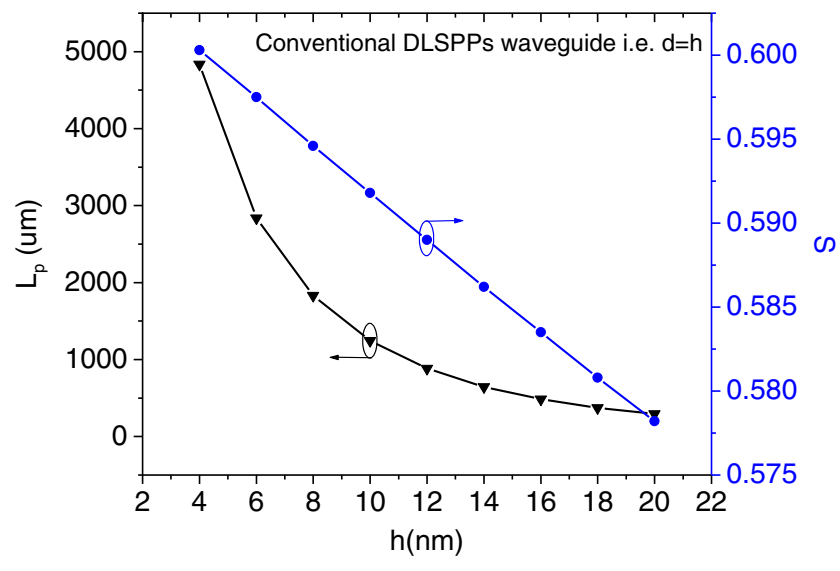

Fig. 2 (Color online) The propagation length $L_{p}$ and normalized mode area $S$ versus thickness $h$ of the metal film. In this case, $H=400 \mathrm{~nm}, G$ $=600 \mathrm{~nm}$ degree of mode confinement (or alternatively, a tighter field confinement for the same propagation length), a patterned metal film thickness structure is proposed, where $d \neq h$ in Fig. 1, and is analyzed.

In our simulation, two sets of fixed parameters are used for our proposed structure: $H=400 \mathrm{~nm}, G=600 \mathrm{~nm}, h=$ $4 \mathrm{~nm}, g=300 \mathrm{~nm}$ and $H=400 \mathrm{~nm}, G=600 \mathrm{~nm}, h=10 \mathrm{~nm}$, $g=300 \mathrm{~nm}$, respectively, corresponding to typical values for such dimensions commonly used by other authors to satisfy the requirements of highly integrated photonic devices needing both tight mode field confinement and long propagation length [11]. Figure 3 shows simulated results of the dependence of the hybrid LRSPPs mode of the proposed structure $\left(L_{p}\right.$ and $\left.S\right)$ on the geometric parameter $d$. The results for the conventional structure $(d=h)$ are also provided in Fig. 3 for comparison.

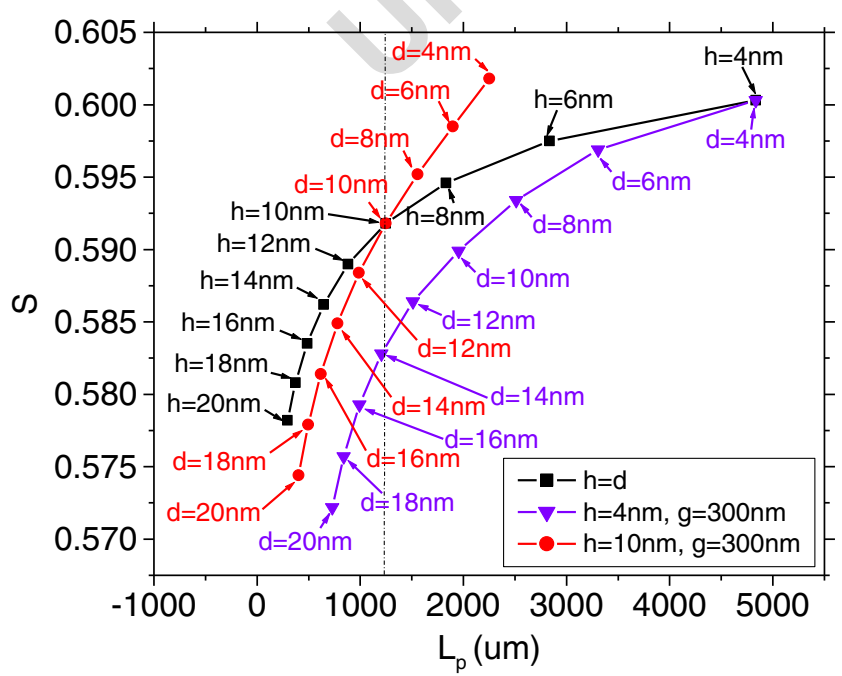

Fig. 3 (Color online) The normalized mode area $S$ as a function of the propagation length $L_{p}$ for the conventional and proposed structure
From Fig. 3, it can be seen that for the same value of $L_{p}, \quad 156$ the value of $S$ is lower (i.e., better mode confinement) for $\quad 157$ our proposed structure as long as $d>h$. However for the 158 simulation with a larger value of $h(h=10 \mathrm{~nm}$, the red curve 159 in Fig. 3), we find that the advantage of the proposed 160 structure is lower as $h$ increases, and in fact beyond a value $\quad 161$ of $L_{p}=1,246 \mu \mathrm{m}$, the proposed structure has a higher value 162 of $S$ (i.e., worse mode confinement).

An alternative view is that for the same value of $S$, the value of $L_{p}$ is higher for our proposed structure while $d>h$. For example for $S=0.58$, the value of $L_{p}$ is $1037 \mu \mathrm{m}$ (violet curve in Fig. 3) for our proposed structure, which is far longer than that of $349 \mu \mathrm{m}$ for the conventional structure. However, for the simulation with a larger value of $h$ (red curve in Fig. 3), it is found that the merit of the proposed structure is reduced as $h$ increases, i.e., for $h=10 \mathrm{~nm}$, at $S=$ 0.58 , the value of $L_{p}$ is only marginally higher that for the conventional structure. Also, we know that beyond a value of $S=0.5918$, the proposed structure actually has a lower value of $L_{p}$.

In addition, the influence of the dimension $g$ on the hybrid LRSPPs mode of the proposed structure $\left(L_{p}\right.$ and $\left.S\right)$ was investigated and simulation results are as shown in Fig. 4. In this simulation, two sets of parameters were selected with $H=400 \mathrm{~nm}, G=600 \mathrm{~nm}, h=4 \mathrm{~nm}, d=20 \mathrm{~nm}$ and $H=400 \mathrm{~nm}, G=600 \mathrm{~nm}, h=10 \mathrm{~nm}, d=20 \mathrm{~nm}$. The corresponding results for the conventional structure are also provided in Fig. 4 for comparison.

As shown in Fig. 4, the performance of the hybrid LRSPPs mode is highly dependent on the value of $g$. The tightest mode confinements can be achieved at $g=300 \mathrm{~nm}$ for both values of h selected. As in Fig. 3, a lower value of $h$ is better. For example, for the same value of $S=0.578$, the

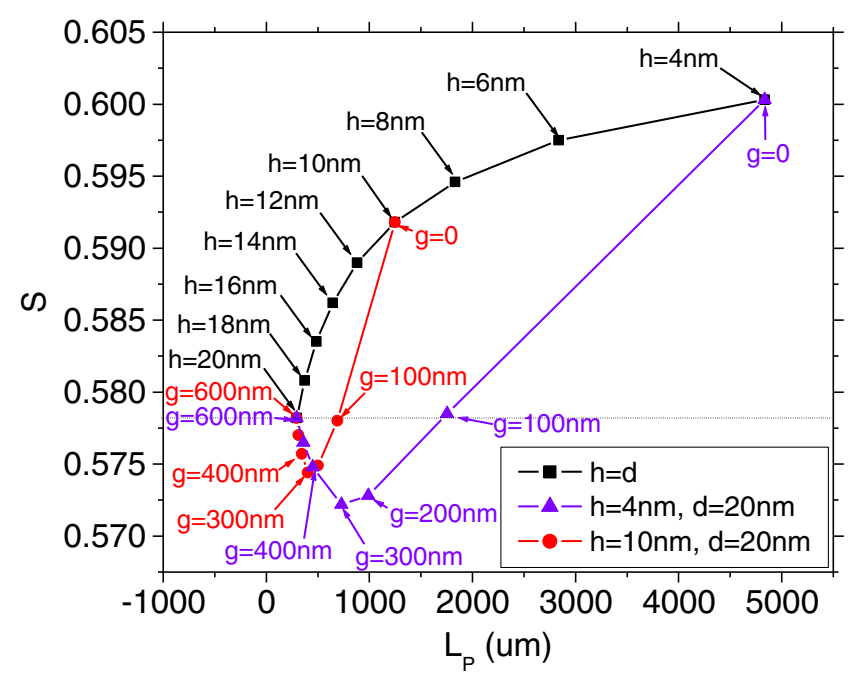

Fig. 4 (Color online) The normalized mode area $S$ versus the propagation length $L_{p}$ for the conventional and proposed structure 
structure with $h=4 \mathrm{~nm}, g=100 \mathrm{~nm}$, and $d=20 \mathrm{~nm}$ has a propagation length of $L p=1753 \mu \mathrm{m}$ compared to a value of $L p=689 \mu \mathrm{m}$ for the structure with $h=10 \mathrm{~nm}, g=100 \mathrm{~nm}$, and $d=20 \mathrm{~nm}$. It should be noted that both proposed structures have substantially longer propagation lengths than of the value of $295 \mu \mathrm{m}$ for the conventional structure.

Based on the results shown in Figs. 3 and 4, we have shown that the proposed DLSPPs waveguide offers a degree of flexibility in the design of SPPs that can achieve both a long propagation length and tight mode confinement simply by appropriately selecting the parameters $g, h$, and $d$.

In future optical communications systems, in order to achieve high-integration density, it is essential to develop ultracompact photonic devices and subsystems. However, photonic subsystems with a high integration density are restricted by the crosstalk between the adjacent waveguides, as well as the parameter of coupling length.

Figure 5a shows the calculated coupling length $L_{\mathrm{c}}$ as a function of separation distance $D$ for two parallel waveguides for different configurations: two horizontally parallel waveguides and two vertically parallel waveguides. The parameters used in the simulation are set as $H=400 \mathrm{~nm}, G$ $=600 \mathrm{~nm}, h=10 \mathrm{~nm}, d=20 \mathrm{~nm}$, and $g=300 \mathrm{~nm}$. For comparison purposes, the coupling lengths for the conventional structures with $d=h=10 \mathrm{~nm}$ was also provided in Fig. 5a. The coupling length is given by $L_{\mathrm{c}}=\pi /\left(\beta_{\mathrm{s}}-\beta_{\mathrm{a}}\right)$, where $\beta_{\mathrm{s}}$ and $\beta_{\mathrm{a}}$ are the propagation constants of the symmetric and anti-symmetric modes of the two adjacent waveguides, respectively. Figure $5 \mathrm{~b}-\mathrm{e}$ are the simulated amplitude distributions of $E_{\mathrm{y}}$ based on our proposed structure. Figure $5 \mathrm{~b}$ and $\mathrm{c}$ show the amplitude distributions of $E_{\mathrm{y}}$ for symmetric and anti-symmetric modes with $D=200 \mathrm{~nm}$ between two horizontally parallel waveguides, and the corresponding results for two vertically parallel waveguides are shown in Fig. 5d and e, respectively.

From Fig. 5a, it can be seen that for both a conventional structure and our proposed structure, the coupling lengths increase almost exponentially for both cases as $D$ increases. However, for the case of vertically parallel waveguides, the coupling length of our proposed structure is higher than that of conventional structure, which illustrates that the crosstalk is lower, in other words, high-density independent function photonic elements are more easily integrated for our proposed structure. For the case of a horizontally parallel waveguide, our proposed structure has similar coupling lengths compared to a conventional structure. Furthermore, for both our proposed structure and a conventional structure it is noted that: (1) the coupling length for the vertically parallel waveguides is higher than that for horizontally parallel waveguides at the same waveguide's separation, which indicates that highly integrated photonic elements are more easily achieved for the vertical configuration; (2) the coupling length for the horizontally parallel waveguides is
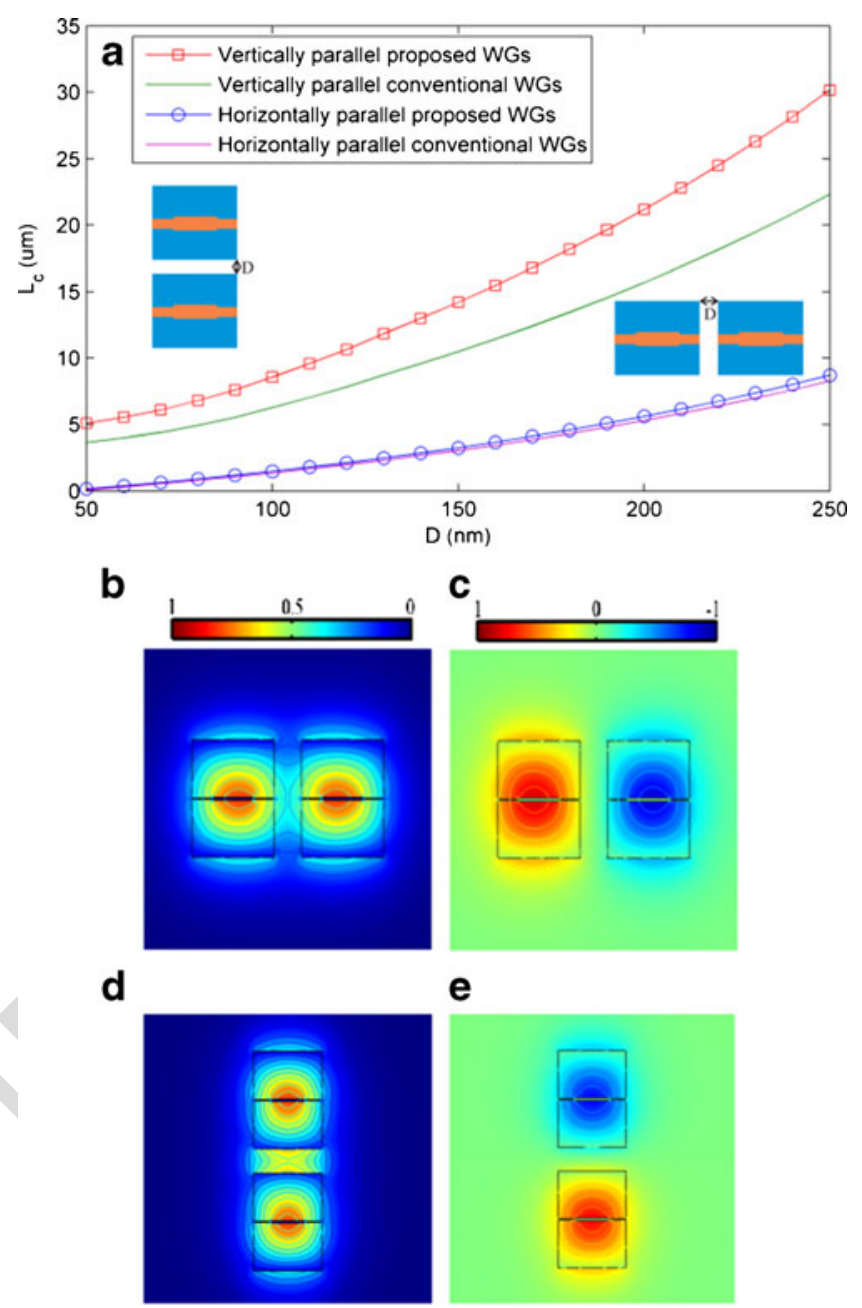

Fig. 5 (Color online) a The calculated coupling lengths versus the separation between the two horizontally and vertically parallel hybrid plasmonic waveguides. Amplitudes of $E_{y}$ for the symmetric mode (b) and the anti-symmetric mode (c) with $D=200 \mathrm{~nm}$ between two horizontally parallel waveguides based on our proposed structure. Amplitudes of $E_{y}$ for the symmetric mode (d) and the anti-symmetric mode (e) with $D=200 \mathrm{~nm}$ between two vertically parallel waveguides based on our proposed structure

significantly lower than that of SPPs waveguide reported in [16], which is typically $10 \mu \mathrm{m}$.

In conclusion, we have proposed a novel dielectricloaded plasmonic waveguide structure which supports hybrid LRSPPs with a high degree of mode confinement and long propagation length. Our investigations show that the proposed structure provides a better trade-off between the propagation length and mode confinement, compared to the conventional DLSPPs configuration. The proposed structure can significantly improve the propagation length for the same mode confinement capacity, or can realize a much tighter field confinement for the same propagation length. The structure offers flexible solutions for the development of compact hybrid plasmonic devices by the appropriate selection of structural parameters. 
Plasmonics

\section{References}

Acknowledgments This work was supported by Dublin Institute of Technology under the Fiosraigh Research Scholarship, Science Foundation Ireland under grant no. 11/TIDA/B2051, 07/SK/I1200, 07/SK/ I1200-STTF11, and the Open Fund of the State Key Laboratory of Information Photonics and Optical Communications (Beijing University of Posts and Telecommunications), China.

1. Kirchain R, Kimerling L (2007) A roadmap for nanophotonics. Nat Photon 1:303-305

2. Barnes WL, Dereux A, Ebbesen T (2003) Surface plasmon subwavelength optics. Nature 424:824-830

3. Chiu NF, Lee JH, Kuan CH, Wu KC, Lee CK, Lin CW (2007) Enhanced luminescence of organic/metal nanostructure for grating coupler active long-range surface plasmonic device. Appl Phys Lett 91:083114

4. Li Q, Song Y, Zhou G, Su YK, Qiu M (2010) Asymmetric plasmonic-dielectric coupler with short coupling length, high extinction ratio, and low insertion loss. Opt Lett 35:3153-3155

5. Han Z, Elezzabi AY, Van V (2010) Experimental realization of subwavelength plasmonic slot waveguides on a silicon platform. Opt Lett 35:502-504

6. Bozhevolnyi SI, Volkov VS, Devaux E, Laluet JY, Ebbesen TW (2006) Channel plasmon subwavelength waveguide components including interferometers and ring resonators. Nature 440:508-511
7. Krasavin AV, Zayats AV (2011) Guiding light at the nanoscale: numerical optimization of ultrasubwavelength metallic wire plasmonic waveguides. Opt Lett 36:3127-3129

8. Joo YH, Jung MJ, Yoon JW, Song SH, Won HS, Park S, Ju JJ (2008) Long-range surface plasmon polaritons on asymmetric double-electrode structures. Appl Phys Lett 92(161103)

9. Berini P (2007) Long-range surface plasmon-polariton waveguides in silica. J Appl Phys 102:053105

10. Zia R, Selker MD, Catrysse PB, Brongersma ML (2004) Geometries and materials for subwavelength surface plasmon modes. J Opt Soc Am A 21:2442-2446

11. Chen JJ, Li Z, Yue S, Gong QH (2009) Hybrid long-range surface plasmon-polariton modes with tight field confinement guided by asymmetrical waveguides. Opt Express 17:23603-23609

12. Tobias H, Jacek G, Sergey IB (2010) Long-range dielectric-loaded surface plasmon-polariton waveguides. Opt Express 18:2300923015

13. Sun XH, Xia LP, Du JL, Yin SY, Du CL (2012) A hybrid longrange surface plasmon waveguide comprising a narrow metal stripe surrounded by the low-index dielectric regions. Opt Commun 285:4359-4363

14. Bao G, Chen ZM, Wu HJ (2005) Adaptive finite-element method for diffraction gratings. JOSA A. 22:1106-1114

15. Oulton RF, Bartal G, Pile DFP, Zhang X (2008) Confinement and propagation characteristics of subwavelength plasmonic modes. New J Phys 10:105018

16. Dai DX, He SL (2009) A silicon-based hybrid plasmonic waveguide with a metal cap for a nano-scale light confinement. Opt Express 17:16646-16653 


\section{AUTHOR QUERY}

AUTHOR PLEASE ANSWER QUERY.

No Query. 